\title{
Associação entre aptidão funcional e excesso de peso em mulheres idosas
}

\author{
Association between functional fitness and overweight in elderly women
}

\author{
C.R. Rech, J.L.S. Cruz, E.D.S. Araújo, F.G. Kalinowski, R.A. Dellagrana
}

RESUMO

O objetivo deste estudo foi verificar associação entre a aptidão funcional (AF) e o excesso de peso (EP) em mulheres idosas. Foram avaliadas 394 mulheres com idade média de 69.4 anos $(D P=3.4$ anos). A AF foi mensurada por meio da bateria de testes motores. O EP foi determinado pela mensuração das variáveis de massa corporal e estatura. Posteriormente foi calculado o índice de massa corporal. Os resultados da aptidão funcional foram comparados com os valores normativos da bateria de testes da AAHPERD para mulheres idosas. Para análise dos dados utilizou-se a análise descritiva. A associação entre a AF e o EP de peso foi verificado pelo teste do Qui-quadrado $(p<.05)$. Os resultados apontam para uma prevalência de EP de $46.5 \%$. Observou-se uma associação estatística $(p<.05)$ entre o índice de AF (IAFG) e o EP entre as idosas, sendo que $87.8 \%$ das idosas com EP apresentaram um IAFG considerado como fraco. Idosas com EP apresentaram 5.07 (IC 95\% $=3.12-7.14$ ) vezes mais probabilidade de terem baixa AF quando comparadas com idosas eutróficas. Os resultados permitem concluir que a condição morfológica (EP) necessita ser considerada na avaliação da AF de mulheres idosas.

Palavras-chave: aptidão funcional, idoso, excesso de peso, testes motores

ABSTRACT The purpose of this study was to verify the association between functional fitness (FF) and excessive fatness (EF) in aged women. Three hundred ninety four women, averaged 69.4 years of age $(D P=3.4$ years), participated in the study. FF was measured by means of a battery of motor tests. EF was determined by corporal mass and height measurements. FF was compared with AAHPERD normative values. Chi-square test $(p<.05)$ was applied in order to verify an association between FF and EF. EF prevalence was $46.5 \%$ and there was an association $(p<.05)$ between the index of functional aptitude (IAFG) and EF. Among those with EF, 87.8\% presented a weak IAFG. EF was associated with a 5.07 (IC $95 \%=3.12-7.14$ ) times greater chance of lower FF. Results point to the conclusion that morphologic condition (EF) needs to be considered in evaluating FF in aged women. Keywords: functional fitness, aged, excessive fatness, motor test

Submetido: 28.12.2009 | Aceite: 25.04.2010

Cassiano Ricardo Rech. Programa de Pós-Graduação em Educação Física da Universidade Federal do Paraná, Brasil. Universidade Estadual de Ponta Grossa, Ponta Grossa, PR, Brasil. Bolsista da Fundação Araucária. Jackson Luis Scarpim Cruz, Eliane Denise da Silveira Araújo e Flávio Guimarães Kalinowski. Universidade Estadual de Ponta Grossa, Ponta Grossa, PR, Brasil.

Rodolfo André Dellagrana. Bolsista da Fundação Araucária e Mestrando da Universidade Federal do Paraná, Curitiba, PR, Brasil.

Endereço para correspondência: Cassiano Ricardo Rech, Rua Dezenove de Dezembro, 344, Apt. 102 - Centro, CEP 84010-390, Ponta Grossa, Paraná - Brasil.

E-mail: crrech@hotmail.com 
O envelhecimento populacional, o excesso de peso e a inatividade física representam problemas de saúde pública emergentes na atualidade. De acordo com a literatura (World Health Organization [WHO], 2002), na Europa, uma em cada três pessoas, no ano de 2050, terá mais de 60 anos de idade. No Brasil, no período correspondente de 1991 a 2000, a população brasileira de idosos teve um crescimento de 4 milhões de pessoas (Instituto Brasileira de Geografia e Estatística [IBGE], 2002).

Da mesma forma que o aumento da população de idosos tem se acentuado, observa-se que a prevalência de obesidade (Barreto, Passos, \& Lima-Costa, 2003; Tavares \& Anjos, 1999) e a inatividade física (Matsudo, Matsudo, \& Barros Neto, 2001; Rosa, Mazo, Silva, \& Brust, 2008) também têm aumentado nesta população. A diminuição dos níveis de atividade física é relatada como um dos fatores que tem contribuído para o aumento da prevalência de excesso de peso em idosos (Nelson et al., 2007). Investigações têm relatado que o excesso de peso e a inatividade física são fatores de risco para o desenvolvimento de doenças cardiovasculares (Da Cruz, Almeida, Schwanke, \& Moriguchi, 2004; Lee, Blair, \& Jackson, 1999; Rexrode, Carey, \& Hennekens, 1998; Sui, Lamonte, \& Laditka, 2007), assim as recomendações para a melhora da qualidade de vida dos idosos têm sido baseadas na elevação dos níveis de atividade física (Arena et al., 2007), pois este fato se reflete na melhora da aptidão funcional de idosos.

A aptidão funcional representa a capacidade do indivíduo em realizar atividades físicas e motoras da vida diária sem dificuldades (Arena et al., 2007). Em idosos, esse conceito tem uma importância extrema devido à possibilidade de proporcionar ao idoso uma independência física para realizar atividades cotidianas.

Neste sentido, a American Alliance for Health, Physical Education, Recreation and Dance (AAHPERD), propôs uma bateria de testes motores específica para idosos, composta por cinco testes motores (coordenação, resistência de força, flexibilidade, agilidade, equilíbrio dinâmico e resistência aeróbia geral), que tem como objetivo avaliar a capacidade física do idoso em realizar tarefas motoras simples (Osness, 1990). Assim, a avaliação da aptidão funcional torna-se necessária para identificar o estado de cada componente específico, como também da aptidão funcional global.

O interesse em analisar a aptidão funcional de idosos reside no fato da mesma demonstrar os efeitos do envelhecimento e também analisar o grau de dependência do idoso para realização destas atividades motoras (Melo \& Giovani, 2004). Nesta população, tem-se estabelecido uma associação entre a baixa aptidão funcional e doenças degenerativas (Maciel \& Guerra, 2008).

O excesso de peso é relacionado com uma baixa aptidão funcional em idosos. Estudos observaram associação entre o excesso de peso e a capacidade de realização de atividades motoras, em idosos fisicamente ativos (Barbosa, Souza, Lebrão, \& Marucci, 2007; Mazo, Kulkpam, Lyra, \& Prado, 2006). Contudo, investigações com idosos, que não realizam atividades físicas, não têm sido relatadas na literatura.

Deste modo, o presente estudo tem como objetivo analisar a associação entre a aptidão funcional e excesso de peso em mulheres idosas não praticantes de atividades físicas regulares.

\section{MÉTODO}

\section{Amostra}

O Município de Ponta Grossa possui uma população de aproximadamente 22885 pessoas. A Fundação Municipal de Promoção ao Idoso (FAPI) possui convênio com 9 entidades, 31 grupos e 4 centros de convivência, totalizando um total de 12000 idosos cadastrados (50 anos ou mais). A amostra selecionada foi obtida entre mulheres 
idosas que não realizavam atividades físicas regulares.

Assim, a população do estudo delimitada para mulheres entre 60 e 79 anos de idade, atendidas pela FAPI no ano de 2008, totalizando uma população de aproximadamente 3560 idosos. Para o tamanho da amostra foi considerada uma prevalência de $40 \%$ de inaptidão funcional (Mazo et al., 2006), com um erro amostral de $3 \%$, deste modo, a amostra mínima foi estimada em 380 indivíduos.

Foram avaliadas 409 idosas, destas foram excluídas 15 idosas devido a não terem concluído a bateria de testes motores. Desta forma, a amostra final foi composta por 394 mulheres idosas, com idade entre 60 e 79 anos, as mesmas foram selecionadas nos grupos de terceira idade da FAPI por meio de convite realizada pelos professores coordenadores dos grupos, sendo que os testes foram aplicados no próprio grupo e em local adequado.

As idosas participantes do estudo foram voluntárias e antes das avaliações as mesmas assinaram um termo de consentimento livre e esclarecido, de acordo com as normas da resolução 196/96 do Conselho Nacional de Saúde e o Estatuto do Idoso (Ministério da Saúde, 2003). O projeto foi aprovado pelo Comitê de Ética em Seres Humanos (protocolo 126/2007).

\section{Procedimentos}

Para determinação da aptidão funcional das idosas foi utilizada a bateria de testes motores da AAHPERD, onde foram avaliadas as variáveis de coordenação motora, força de resistência, flexibilidade, agilidade, equilíbrio dinâmico e a resistência aeróbia geral. Por meio dos escores individuais também foi possível calcular o índice de aptidão funcional geral (IAFG) das idosas, de acordo com a proposta de Osness (1990). Os dados dos testes de aptidão funcional foram comparados com a tabela normativa proposta por Zago e Gobbi (2003) e Benedetti et al. (2007). Os idosos foram classificados em duas categorias: Fraco (muito fraco e fraco) e Bom (regular, bom e muito bom).

A massa corporal foi mensurada por meio de uma balança Plenna digital com capacidade de $150 \mathrm{~kg}$ e resolução de 100 gramas, a estatura foi mensurada por meio de uma fita métrica fixada à parede com precisão de $.1 \mathrm{~cm}$ de acordo com os procedimentos descritos por Alvarez e Pavan (2007). A partir destas medidas foi calculado o índice de massa corporal (IMC) pela razão entre a massa corporal e a estatura ao quadrado. As idosas foram classificadas em relação ao excesso de peso de acordo com as padronizações proposta por Lipschitz (1994), onde o IMC igual ou acima de $28 \mathrm{~kg} / \mathrm{m}^{2}$ foi caracterizado como excesso de peso e abaixo de $28 \mathrm{~kg} / \mathrm{m}^{2}$ como eutrófico.

\section{Análise estatística}

Para análise dos dados foram utilizados a análise descritiva (média, desvio padrão e frequências) e o teste do Qui-quadrado (teste exato de Fisher) para realizar a análise estatística dos dados. Para realização dos cálculos estatísticos foram utilizados os programas do Excel e o SPSS 10.0 para Windows.

\section{RESULTADOS}

O presente estudo objetivou investigar a associação entre o índice de aptidão funcional (IAFG) e o excesso de peso em mulheres idosas atendidas por grupos de terceira idade da cidade de Ponta Grossa, Paraná. Foram avaliadas 394 mulheres com idade entre 60 e 79 anos. A tabela 1 apresenta características físicas nas mulheres idosas que participaram do estudo.

Para a composição do IAFG foi utilizada a bateria de testes motores proposta pela AAHPERD e foram adotados 5 testes motores. A tabela 2 apresenta os valores médios e desvios padrão obtidos pelas idosas em cada teste. 
Tabela 1

Valores descritivos de média (M), desvio padrão (DP) e amplitude para as variáveis descritivas da amostra $(n=394)$ de mulheres idosas, Ponta Grossa, 2008

\begin{tabular}{ccccc}
\hline Variável & M & DP & Mínimo & Máximo \\
\hline Idade & 69.4 & 3.4 & 60 & 79 \\
Massa corporal $(\mathrm{kg})$ & 67.6 & 8.9 & 43.0 & 96.3 \\
Estatura $(\mathrm{cm})$ & 153.6 & 7.4 & 136 & 173 \\
IMC $\left(\mathrm{kg} / \mathrm{m}^{2}\right)$ & 28.5 & 4.6 & 20.8 & 37.1 \\
\hline
\end{tabular}

Tabela 2

Valores descritivos de média (M), desvio padrão (DP) e amplitude dos testes de aptidão funcional de mulheres idosas, Ponta Grossa, 2008

\begin{tabular}{ccccc}
\hline Teste Motor & M & DP & Mínimo & Máximo \\
\hline Agilidade (s) & 30.1 & 4.7 & 23 & 44 \\
Coordenação (s) & 15.9 & 3.8 & 8.3 & 33.0 \\
Flexibilidade (cm) & 51.2 & 11.8 & 13.0 & 79.5 \\
Força (rep) & 17.3 & 4.3 & 11 & 33 \\
Resistência aeróbica (s) & 548.6 & 72.3 & 435 & 756 \\
\hline
\end{tabular}

Os resultados da amplitude dos dados (ver tabela 2) indicam que existe uma heterogeneidade nos escores das idosas que participaram do estudo, em especial nos testes de coordenação, flexibilidade e força.

Com relação ao estado nutricional observou-se que $53.5 \%$ da amostra são eutróficos, demonstrando uma elevada prevalência de excesso de peso $(46.5 \%)$ e, vale ressaltar que não foi encontrado nenhum caso de baixo peso na amostra.

A tabela 3 apresenta os valores absolutos e relativos das frequências dos testes motores da aptidão funcional das idosas da amostra. A maioria das idosas avaliadas (77.6\%) apresentou níveis da aptidão funcional abaixo dos níveis mínimos estabelecidos por Gobbi e Zago (2003) e Benedetti et al. (2007). Esse baixo nível se acentua em especial nas variáveis físicas da agilidade, coordenação e resistência, respectivamente. A variável física de melhor desempenho alcançado foi a da flexibilidade, onde $41.1 \%$ da amostra obtiveram índices dentro das classificações bom e muito bom, seguida pela força (18.8\%) e resistência (18.8\%).

Tabela 3

Frequência relativa (\%) do índice de aptidão funcional de mulheres idosas, Ponta Grossa, 2008

\begin{tabular}{cccccc}
\hline Testes motores & Muito fraco & Fraco & Regular & Bom & Muito bom \\
\hline Agilidade (s) & 72.9 & 16.5 & 8.2 & 1.2 & 1.2 \\
Coordenação (s) & 57.6 & 25.9 & 11.8 & 3.5 & 1.2 \\
Flexibilidade (cm) & 4.7 & 30.6 & 23.5 & 23.5 & 17.7 \\
Força (rep) & 40.0 & 24.7 & 16.5 & 15.3 & 3.5 \\
Resistência aeróbica (s) & 42.4 & 29.4 & 9.4 & 9.4 & 9.4 \\
IAFG & 24.7 & 52.9 & 18.8 & 3.6 & 0.0 \\
\hline
\end{tabular}

Nota: IAFG = índice de aptidão física geral

Quando foi realizada a análise da associação entre o IAFG e o excesso de peso das idosas (tabela 4), pode ser observado que ocorreu uma associação estatisticamente significativa 
$(p<.05)$. Isso demonstrou que as idosas com excesso de peso (sobrepeso e obesidade) apresentam menores índices de IAFG. Idosas com excesso de peso apresentaram 5.07 (IC $95 \%=3.12-7.14)$ vezes mais probabilidades de terem baixa aptidão funcional quando comparadas com idosas eutróficas.

Tabela 4

Associação entre o indice de aptidão física geral (IAFG) e o estado nutricional de aptidão funcional de mulheres idosas $(n=394)$, Ponta Grossa, 2008

\begin{tabular}{cccc}
\hline \multirow{2}{*}{ Nível IAFG } & \multicolumn{2}{c}{ Estado nutricional } & \multirow{2}{*}{$p$} \\
\cline { 2 - 3 } & Eutrófico & Excesso peso & \\
\hline Bom & $80.6 \%$ & $12.2 \%$ & \multirow{2}{*}{001} \\
Fraco & $19.4 \%$ & $87.8 \% *$ & \\
\hline Total & $100.0 \%$ & $100.0 \%$ & \\
\hline
\end{tabular}

${ }^{*} p<.05$, associação significativa (teste exato de Fisher);

Fraco: muito fraco e fraco; Bom: regular, bom e muito bom

\section{DISCUSSÃO}

O acelerado envelhecimento populacional é um fenômeno observado tanto em países desenvolvidos como nos países em desenvolvimento (Carvalho \& RodriguesWong, 2008), este quadro tem proporcionado um aumento no interesse no estudo da relação entre o envelhecimento e fatores que contribuem para uma diminuição da aptidão funcional em idosos.

Evidências científicas têm demonstrado que com o avanço da idade existe uma diminuição da aptidão funcional (Maciel \& Guerra, 2008; Rauen, Moreira, Calvo, \& Lobo, 2008). No presente estudo observou-se que $77.6 \%$ das mulheres avaliadas apresentaram um nível baixo de aptidão funcional e apenas $3.0 \%$ apresentaram uma aptidão funcional considerada como bom (tabela 3).

Estes resultados corroboram com os dados apresentados por outras investigações que apontam para um baixo nível de aptidão física em idosos. Rogatto e Gobbi (2001) observaram uma diminuição da força muscular em idosos, mesmo com a prática regular de atividade física. Alves, Mota, Costa e Alves (2004) relatam que esta diminuição de força está relacionada com uma maior probabilidade de quedas em idosos. Barbosa et al. (2007) observaram que idosas com excesso de peso foram mais lentas na realização dos testes motores. Na amostra estudada, $64.7 \%$ (ver tabela 3) apresentaram um nível de força considerado baixo ou muito baixo.

Mesmo em idosos praticantes de atividade física, Rosa et al. (2008) observaram um baixo IAFG (58.1\%). Esses resultados vão ao encontro dos relatados no presente estudo (ver tabela 3). Além disso, em estudo com acompanhamento de 12 anos, idosas com prática regular de atividade física demonstraram melhoras significativas em relação ao grupo controle para as variáveis de coordenação, flexibilidade e agilidade, porém, o grupo de intervenção apresentou melhores valores pré-teste para estas variáveis, principalmente para a flexibilidade (Pauli, Souza, Zago, \& Gobbi, 2009), desta forma, nota-se a dificuldade na manutenção da aptidão funcional em idosas.

Quanto ao estado nutricional nota-se que $46.5 \%$ da amostra apresentou excesso de peso, estes resultados são similares aos apresentados por levantamentos nacionais no grupo de idosos que vem apontando para uma elevada prevalência de excesso de peso nesta população (Barbosa et al., 2007; Tavares \& Anjos, 1999). Da mesma maneira, Lebrão e Laurenti (2005) encontraram elevada prevalência de excesso de peso em mulheres idosas (40.5\%).

A maior prevalência de excesso de peso é preocupante uma vez que está diretamente relacionada com uma menor capacidade funcional em idosas fisicamente ativas (Barbosa et al., 2007; Mazo et al., 2006), maior relato de casos de depressão (Moraes et al., 2007), e maior chance de morte (Maciel \& Guerra, 2008; Sui et al., 2007). Além da maior chance de desenvolver hipertensão arterial, diabetes mellitus, hipertrigliceridemia e doenças cardíacas (Da Cruz et al., 2004; Rexrode et al., 1998).

Houve uma associação estatística $(p<.05)$ entre o IAFG e o excesso de peso no presente estudo, demonstrando que mulheres idosas 
que apresentam excesso de peso tem uma maior prevalência de baixa aptidão funcional.

Verificou-se que $87.8 \%$ das idosas com excesso de peso apresentaram um baixo nível de aptidão funcional. Em estudo com idosas, Mazo et al. (2006) observou que $90.4 \%$ das idosas com um IAFG classificado como fraco, apresentaram excesso de peso. Assim como Barbosa et al. (2007) que verificou a associação entre o desempenho motor e o IMC, sendo que $14.4 \%$ das idosas com idade entre 60 e 69 anos e com excesso de peso, apresentaram um resultado fraco dentro do desempenho motor. No mesmo estudo, os autores observaram que $29.3 \%$ das idosas com idade entre 70 e 79 anos e excesso de peso apresentaram um desempenho motor fraco.

Assim, nota-se que um programa de promoção da saúde em idosos necessita considerar a relação entre o nível de aptidão funcional e o excesso de peso corporal. Pois os achados do presente estudo apontam para uma tendência que é visualizada em outras regiões do país e que tem demonstrado que o acúmulo de gordura corporal está associado com a diminuição da aptidão funcional em idosas (Instituto do Câncer [INCA], 2004).

O presente estudo não contou com a participação de pessoas do gênero masculino, em virtude do baixo número de idosos cadastrados nos grupos de terceira idade e também pelo fato de ainda não existir uma tabela normativa dos testes motores para pessoas do gênero masculino.

Outra limitação encontrada foi que algumas idosas não conseguiram realizar alguns dos testes motores, devido a alguns problemas de saúde, como artrose, doença de Parkinson e até mesmo insuficiência respiratória, o que impossibilitou a realização dos testes.

\section{CONCLUSÕES}

Os resultados do presente estudo mostram que ocorre uma associação inversa entre o IAFG e o IMC das idosas, ou seja, idosas com níveis mais baixos do IAFG apresentam um IMC classificado como excesso de peso. Assim, a manutenção da aptidão funcional e níveis normais de IMC podem ser fundamentais para melhorar a qualidade de vida de idosas.

\section{REFERÊNCIAS}

Alvarez, B. R., \& Pavan, A. L. (2007). Alturas e comprimentos. In E. L. Petroski (Ed.), Antropometria: Técnicas e padronizações (pp. 3845). Porto Alegre: Palloti.

Alves, R. V., Mota, J., Costa, M. C., \& Alves, J. G. B. (2004). Aptidão física relacionada à saúde de idosos: Influência da hidroginástica. Revista Brasileira de Medicina do Esporte, 10(1), 31-37.

Arena, R., Myers, J., Williams, M. A., Gulati, M., Kligfiel, P. J., Balady, G. J., et al. (2007). Assessment of functional capacity in clinical and research settings: A scientific statement from the American Heart Association Committee on Exercise, Rehabilitation, and Prevention of the Council on Clinical Cardiology and the Council on Cardiovascular Nursing. Circulation, 116, 329-343.

Barbosa, A. R., Souza, J. M. P., Lebrão, M. L., \& Marucci, M. F. N. (2007). Estado nutricional e desempenho motor de idosos de São Paulo. Revista da Associação Médica Brasileira, 53(1), 7579.

Barreto, S. M., Passos, V. M. A., \& Lima-Costa, M. F. F. (2003). Obesidade e baixo peso entre idosos brasileiros: Projeto Bambuí. Cadernos de Saúde Pública, 19(2), 605-612.

Benedetti, T. R. B., Mazo, G. Z., Gobbi, S., Amorim, M., Gobbi, L. T. B., Ferreira, L., et al. (2007). Valores normativos de aptidão funcional em mulheres de 70 a 79 anos. Revista Brasileira de Cineantropometria e Desempenho Humano, 9(1), 28-36.

Carvalho, J., \& Rodrigues-Wong, L. L. (2008). A transição da estrutura etária da população brasileira na primeira metade do século XXI. Cadernos de Saúde Pública, 24(3), 597-560.

Da Cruz, I. B. M., Almeida, M. S. C., Schwanke, H. A., \& Moriguchi, C. H. (2004). Prevalência de obesidade em idosos longevos e sua associação com fatores de risco e morbidades cardiovasculares. Revista da Associação Médica Brasileira, 50(2), 172-177.

Instituto Brasileiro de Geografia e Estatística - IBGE (2002). Perfil dos idosos responsáveis pelos domicílios no Brasil, 2000. Rio de Janeiro: Autor.

Instituto do Câncer (2004). Inquérito domiciliar sobre comportamentos de risco e morbidade referida de 
doenças e agravos não transmissíveis: Brasil, 15 capitais e Distrito Federal, 2002-2003. Consultado em 12 de Outubro de 2009, a partir de http://www.inca.gov.br/inquerito/

Lebrão, M. L., \& Laurenti, R. (2005). Saúde, bemestar e envelhecimento: O estudo SABE no Município de São Paulo. Revista Brasileira de Epidemiologia, 8(2), 127-141.

Lee, C. D., Blair, S. N., \& Jackson, A. S. (1999). Cardiorespiratory fitness, body composition, and all-cause and cardiovascular disease mortality in men. American Journal of Clinical Nutrition, 69(3), 373-380.

Lipschitz, D. A. (1994). Screening for nutritional status in the elderly. Primary Care, 21 (1), 55-67.

Maciel, A. C. C., \& Guerra, R. O. (2008). Limitação funcional e sobrevida em idosos de comunidade. Revista da Associação Médica Brasileira, 54(4), 347-352.

Matsudo, S. M., Matsudo, V. K. R., \& Barros Neto, T. L. (2001). Atividade física envelhecimento: Aspectos epidemiológicos. Revista Brasileira de Medicina do Esporte, 7(1), 2-13.

Mazo, G. Z., Kulkpam, W., Lyra, V. B., \& Prado, A. P. M. (2006). Aptidão funcional geral e índice de massa corporal de idosas praticantes de atividade física. Revista Brasileira de Cineantropomotria e Desempenho Humano, 8(4), 46-51.

Melo, G. F., \& Giavoni, A. (2004). Comparação dos efeitos da ginástica aeróbica e da hidroginástica na comparação corporal de mulheres idosas. Revista Brasileira de Ciência e Movimento, 12 (2), 13-18.

Ministério da Saúde (2003). Estatuto do idoso (2a ed.). Brasília: Editora MS.

Moraes, H., Deslandes, A., Ferreira, C., Pompeu, F. A. M. S., Ribeiro, P., \& Larks, J. (2007). O exercício físico no tratamento da depressão em idosos: Revisão sistemática. Revista de Psiquiatria, 29(1), 70-79.

Nelson, M. E., Rejeski, W. J., Blair, S. N., Duncan, P. W., Judge, J. O., King, A. C., et al. (2007). Physical activity and public health in older adults: Recommendation from the American College of Sports Medicine and the American Heart Association. Circulation, 116, 1094-1105.
Osness, W. H. (1990). Functional fitness assessment for adults over 60 years. Reston: American Alliance for Health, Physical Education, Recreation and Dance.

Pauli, J. R., Souza, L. S., Zago, A. S., \& Gobbi, S. (2009). Influência de 12 anos de prática de atividade física regular em programa supervisionado para idosos. Revista Brasileira de Cineantropometria e Desempenho Humano, 11 (3), 255-260.

Rauen, M. S., Moreira, E. A. M., Calvo, M. C. M., \& Lobo, A. S. (2008). Avaliação do estado nutricional de idosos institucionalizados. Revista de Nutrição, 21 (3), 303-310.

Rexrode, K. M., Carey, V. J., \& Hennekens, C. H. (1998). Abdominal adiposity and coronary heart disease in women. The Journal of the American Medical Association, 280(21), 18431848.

Rogatto, G. P., \& Gobbi, S. (2001). Efeitos da atividade física regular sobre parâmetros antropométricos e funcionais de mulheres jovens e idosas. Revista Brasileira de Cineantropometria e Desempenho Humano, 3(1), 63-69.

Rosa, M. F., Mazo, G. Z., Silva, A. H., \& Brust, C. (2008). Efeito do período de interrupção de atividades aquáticas na aptidão funcional de idosas. Revista Brasileira de Cineantropometria e Desempenho Humano, 10(3), 237-242.

Sui, X., Lamonte, M. J., \& Laditka, J. N. (2007). Cardiorespiratory fitness and adiposity as mortality predictors in older adults. The Journal of the American Medical Association, 298(21), 2507-2516.

Tavares, E. L., \& Anjos, L. A. (1999). Perfil antropométrico da população brasileira: Resultados da pesquisa nacional sobre saúde e nutrição. Cadernos de Saúde Pública, 15(4), 759768.

World Health Organization (2002). Active ageing: A policy framework. Geneva: Autor.

Zago, A. S., \& Gobbi, S. (2003). Valores normativos da aptidão funcional de mulheres de 60 a 70 anos. Revista Brasileira de Ciência e Movimento, 11 (2), 77-86. 